\title{
Introduction to the Special Issue on USENIX FAST 2017
}

This special issue of the ACM Transactions on Storage presents some of the highlights of the 15th USENIX Conference on File and Storage Technologies (FAST'17). In the 15 years since its inception, FAST has grown into a damn active community, attracting 116 submissions and over 400 attendees in 2017. FAST'17 continues the tradition of bringing together storage-system researchers and practitioners to explore new directions in the design, implementation, evaluation, and deployment of storage systems. FAST takes a broad view of storage systems, encompassing everything from lowlevel storage devices to information management systems. We selected five high-quality articles for publication in this special issue of ACM Transactions on Storage.

The first article, which was also selected as one of the best papers at the conference, is "Application Crash Consistency and Performance with CCFS," by Thanumalayan Sankaranarayana Pillai, Ramnatthan Alagappan, Lanyue Lu, Vijay Chidambaram, Andrea C. Arpaci-Dusseau, and Remzi H. Arpaci-Dusseau. This article introduces a crash-consistent file system that uses a stream abstraction to guarantee that intra-stream operations are committed in program order to improve the correctness of application crash consistency, while removing ordering constraints across streams to maintain high performance.

The second article is "Redundancy Does Not Imply Fault Tolerance: Analysis of Distributed Storage Reactions to File-System Faults," by Aishwarya Ganesan, Ramnatthan Alagappan, Andrea C. Arpaci-Dusseau, and Remzi H. Arpaci-Dusseau. The authors analyze eight popular distributed storage systems and uncover significant problems that can result in data loss, corruption, and unavailability, with implications for the design of future fault-tolerant distributed systems.

The third article is "vNFS: Maximizing NFS Performance with Compounds and Vectorized I/O," by Ming Chen, Geetika Babu Bangera, Dean Hildebrand, Farhaan Jalia, Geoff Kuenning, Henry Nelson, and Erez Zadok. This article leverages the NFSv4 compounding feature, exposing a highlevel vectorized API to support bulk operations, achieving dramatic speedups on a wide range of workloads under various network latency conditions.

The fourth article is "Tiny-Tail Flash: Near-Perfect Elimination of Garbage Collection Tail Latencies in NAND SSDs," by Shiqin Yan, Huaicheng Li, Mingzhe Hao, Michael Hao Tong, Swaminathan Sundararaman, Andrew A. Chien, and Haryadi S. Gunawi. A well-known problem with flash storage is the issue of long, unpredictable delays induced by garbage collection in the flash translation layer. The authors use a combination of novel strategies to optimize garbage collection, achieving tail latencies that approach the ideal of a system without garbage collection.

The final article, also selected as one of the best papers at the conference, is "Efficient Free Space Reclamation in WAFL," by Ram Kesavan, Rohit Singh, Travis Grusecki, and Yuvraj Patel. The NetApp WAFL file system uses copy-on-write for high-performance writes and efficient snapshots, increasing the demand for free-space reclamation. The authors present the evolution of algorithms and data structures spanning more than a decade of production deployments.

Geoff Kuenning and Carl Waldspurger Program Committee Co-Chairs

USENIX FAST 2017

2017 Copyright is held by the owner/author(s).

1553-3077/2017/08-ART18

https://doi.org/10.1145/3131620

ACM Transactions on Storage, Vol. 13, No. 3, Article 18. Publication date: August 2017. 